\title{
Data mining and visualization from planetary missions: the VESPA-Europlanet2020 activity
}

\author{
Andrea Longobardo ${ }^{1}$, Maria Teresa Capria ${ }^{1,2}$, Angelo Zinzi ${ }^{2,3}$, \\ Stavro Ivanovski ${ }^{1}$, Marco Giardino ${ }^{1}$, Giuseppe Di Persio ${ }^{1}$, \\ Sergio Fonte ${ }^{1}$, Ernesto Palomba ${ }^{1,2}$, Lucio Angelo Antonelli ${ }^{2,3}$, \\ Sergio Fonte ${ }^{1}$, Paolo Giommi \\ and the Europlanet VESPA 2020 Team \\ ${ }^{1}$ INAF-IAPS, via del Fosso del Cavaliere 100, 00133, Rome, Italy \\ email: andrea.longobardo@iaps.inaf.it \\ ${ }^{2}$ ASI-ASDC, via del Politecnico snc, 00133, Rome, Italy \\ ${ }^{3}$ INAF-OAR, via di Frascati 33, 00040 Monte Porzio Catone (RM), Italy
}

\begin{abstract}
This paper presents the VESPA (Virtual European Solar and Planetary Access) activity, developed in the context of the Europlanet 2020 Horizon project, aimed at providing tools for analysis and visualization of planetary data provided by space missions. In particular, the activity is focused on minor bodies of the Solar System. The structure of the computation node, the algorithms developed for analysis of planetary surfaces and cometary comae and the tools for data visualization are presented.
\end{abstract}

Keywords. methods: data analysis, minor planets, asteroids

\section{Introduction}

The Europlanet 2020 Research Infrastructure is a Horizon 2020 project aimed at integrating and supporting planetary science across Europe. The project is led by the Open University, UK, and has 33 beneficiary institutions from 19 European countries.

VESPA (Virtual European Solar and Planetary Access) is an activity developed in the framework of the Europlanet2020 project, aimed at building a Virtual Observatory (VO) for Planetary Science. This VO will contain and connect every sort of planetary data, and will provide tools aimed at retrieving, displaying and correlating data and results obtained from their analysis.

Currently, by means of the protocols of the VO, we are making available the data of the Vesta and Ceres asteroids provided from the NASA/Dawn mission, in particular from the VIR spectrometer (De Sanctis, M.C. et al. (2011)). VESPA will make it possible to perform basic analysis of these data. First, it will increase the accessibility, by allowing the scientists to retrieve data directly from the web portal. Second, it will maximize their usability, by improving the collaboration between different virtual observatory tools, allowing the user to share data sets, taking advantage of the specific features of each tool. The structure of the Europlanet 2020 computation node (EPN - TAP) is explained in Section 2, whereas Section 3 will be devoted to tools already developed or under development for visualization and analysis of planetary data. Finally, in Section 4 the next steps will be identified. 


\section{The EPN - TAP node}

In the context of the VESPA framework, we are currently developing an EPN-TAP service node.

This node is based on the Gavo DaCHS, a software platform, based on Python, suitable for implementing TAP services. The plan is to realize a new single access point for different original datasets produced by our Institute, unifying the access mechanism under the same technology, adopting the virtual observatory approach. Being compliant to the EPN-TAP protocol version 2, will make our datasets reachable through the VESPA client and its query interface (http://vespa.obspm.fr/planetary/data/epn/query/all/), greatly improving their exposure by several orders of magnitude. On the other side, compliance with the IVOA VO formats will make it possible to reach the full interoperability with popular plotting tools, through the SAMP protocol. The currently selected datasets are:

- Dust catalog of interplanetary dust particles (1424 particles);

- Catalog of cometary emission lines (33183 lines);

- VIS and NIR spectra from Dawn/VIR spectrometer;

- Magnetohydrodynamic instabilities model outputs applied to cometary environment.

\section{Tools}

Here we will show the tools developed and under development for the analysis of minor bodies of the Solar System. The algorithms will allow the analysis of both surface and cometary comas of these bodies, whereas the MATISSE tool will allow 3D visualization of data.

\subsection{Algorithms for the analysis of planetary surfaces}

The algorithms for the analysis of planetary surfaces are based on those developed for the analysis of Vesta data (Longobardo, A. et al. (2014)) provided by the imaging spectrometer VIR onboard the Dawn spacecraft (Russell, C. T. et al. (2012)). In particular, the following operations will be available:

- Conversion from reflected radiance to surface reflectance, by taking into account the heliocentric distance;

- Despiking, by recognizing and removing the spikes on the spectra, due to noise;

- Bridging visible and infrared spectra, by defining the overlapping spectral region;

- Retrieval reflectance at selected wavelengths, by selecting the relative spectrometer channel and maximizing the Signal-to-Noise ratio by means of averaging operations;

- Photometric correction, by considering an appropriate disk function to remove topography effects and by calculating the phase function by means of statistical analysis;

- Retrieval of absorption bands, by defining the band shoulders and troughs by means of polynomial fits, the continuum by means of a linear fit, and, consequently, the band depth, minimum and center (i.e. the minimum after continuum removal);

- Retrieval of spectral indices, such as spectral slopes or combination of reflectances;

- Retrieval of content of specific minerals if this content can be obtained by band center analyses;

- Detection of dark and bright regions (e. g., Palomba, E. et al. (2014)), by means of a statistical analysis on reflectance values.

\subsection{Algorithms for analysis of cometary comae}

The algorithms dealing with cometary comae are based on those developed for the analysis of $67 \mathrm{P} /$ Churyumov-Gerasimenko comet data provided by the VIRTIS spectrometer (Coradini, A. et al. (1997)) and the GIADA dust instrument (Colangeli, L. et al. (1997)) 
onboard the ESA/Rosetta mission. In particular, regarding the cometary dust dynamics, the following services and data analysis are planned to be available:

- Interactive remote access calculation of the aerodynamic coefficients for spherical and aspherical dust grains.

These coefficients are crucial for computing the aerodynamic force acting on ejected cometary dust. The aerodynamic force is one of the driving forces that plays role in the dust dynamics of the circumnuclear coma. The tool will facilitate the users to compute the aerodynamic force acting on grains of simple shapes like ellipsoids of revolutions of different aspect ratio (Ivanovski, S. L. et al. (2017)). Most of the state-of-the-art gas-dust dynamical models use spherical particles. The tool will also supply averaged aerodynamic coefficients for different convex shape bodies that can be used as "refined" values in the spherical dust codes that require computation of the aerodynamic force.

- Interactive dust distribution map views of the released GIADA data.

The GIADA instrument, on-board the Rosetta space probe was devoted to measure momentum, mass, speed and cross section of individual dust particles emitted by comet 67P/Churyumov-Gerasimenko. These maps with the GIADA data will allow views of the 67P/Churyumov-Gerasimenko comet dust particle distribution seen by GIADA, showing the dust particle location, speeds and time evolution during different observational periods of the Rosetta mission.

\subsection{D visualization of data: the MATISSE tool}

Since minor bodies generally present very irregular shapes, c̈lassicalbidimensional projections of observations are difficult to understand. With the aim of providing to the scientific community a tool to access, visualize and analyze data in a new way, ASI Science Data Center started to develop MATISSE (Multi-purposed Advanced Tool for the Instruments for the Solar System Exploration - http://tools.asdc.asi.it/matisse.jsp Zinzi, A. et al. (2016)) in late 2012.

This tool allows 3D web-based visualization of data acquired by planetary exploration missions: the output could either be the straightforward projection of the selected observation over the shape model of the target body or the visualization of a high-order product (average/mosaic, difference, ratio, RGB) computed directly online with MATISSE.

Standard outputs of the tool also comprise downloadable files to be used with GIS software (GeoTIFF and ENVI format) and 3D very high-resolution files to be viewed by means of the free software Paraview.

Up to now the first and most frequent exploitation of the tool has been related to visualization of data acquired by the VIRTIS-M instrument onboard Rosetta that observed the comet $67 \mathrm{P} /$ Churyumov-Gerasimenko. The success of this task, well represented by the good number of published works that used images made with MATISSE, confirmed the need of a different approach to correctly visualize data coming from irregularly-shaped bodies and from their surroundings (e.g., coma and jets).

However, MATISSE usage has not been limited to this mission only, also VIR on Dawn observations of 4 Vesta and 1 Ceres have been included in the available MATISSE datasets. It is also interesting to note that, thanks to its robust capabilities in the production of higher-order products, data from larger objects (such as the Chinese Chang'e 1 and 2 lunar data and Mercury's NASA Messenger ones) are accessible by means of MATISSE. 


\section{Future perspectives}

The algorithms will be tested on different minor bodies data and the obtained results will be merged with the MATISSE tool, in order to allow their 3D visualization. New MATISSE tasks will be added, such as those related to the GIADA-Rosetta observations and those requiring standard protocols to access data stored on external repositories, such as NASA ODE (Bennett, K. J. et al. (2014)) and Planetary VO (Erard, S. et al. (2014)).

In the meanwhile, the EPN-TAP node will be completed in order to provide VO accessibility to each user as final result.

\section{Acknowledgements}

This work has been developed in the framework of the Europlanet 2020 RI, which has received funding from the European Union's Horizon 2020 research and innovation programme under grant agreement No 654208.

\section{References}

Bennett et al. Acessing PDS data in pipeline processing and websites through PDS Geoscience orbital data explorer's web-based API(REST) interface, 2014, 45 th Lunar and Planetary Science Conference 2014, LPI Contribution No. 1777, p.1026

Colangeli, L. et al. GIADA: The Grain Impact Analyser and Dust Accumulator for the Rosetta space mission., 1997 Advances in Space Research, 39, 3, 446-450.

Coradini, A. et al. VIRTIS: the imaging spectrometer of the Rosetta mission., 1997 Advances in Space Research, 24, 9, 1095-1104.

De Sanctis, M. C. et al. The VIR Spectrometer., 2011 Space Science Reviews, 163, 329?369.

Erard, S. et al. Planetary Science Virtual Observatory Architecture, 2014 Astronomy and Computing, 7-8, 71-80, doi:10.1016/j.ascom.2014.07.005

Ivanovski, S. L. et al. Dynamics of aspherical dust grains in a cometary atmosphere: I. axially symmetric grains in a spherically symmetric atmosphere), 2017 Icarus, 282, 333-350

Longobardo, A. et al. Photometric behavior of spectral parameters in Vesta dark and bright regions as inferred by the Dawn VIR spectrometer.), 2014 Icarus, 240, 20-35.

Palomba, E. et al. Composition and mineralogy of dark material units on Vesta, 2014 Icarus, $240,58-72$.

Russell, C. T. et al. Dawn at Vesta: Testing the protoplanetary paradigm, 2012 Science, 336, 6082, 684-686.

Zinzi, A. et al. Dawn at Vesta: Testing the protoplanetary paradigm, 2012 Astronomy and Computing, http://dx.doi.org/10.1016/j.ascom.2016.02.006 